University of Nebraska - Lincoln

DigitalCommons@University of Nebraska - Lincoln

Faculty Publications, Department of Child, Youth, and Family Studies

3-2019

\title{
Adult attachment and testosterone reactivity: Fathers' avoidance predicts changes in testosterone during the strange situation procedure
}

Robin S. Edelstein

Kristi Chin

Ekjyot K. Saini

Patty X. Kuo

Oliver C. Schultheiss

See next page for additional authors

Follow this and additional works at: https://digitalcommons.unl.edu/famconfacpub

Part of the Developmental Psychology Commons, Family, Life Course, and Society Commons, Other Psychology Commons, and the Other Sociology Commons

This Article is brought to you for free and open access by the Child, Youth, and Family Studies, Department of at DigitalCommons@University of Nebraska - Lincoln. It has been accepted for inclusion in Faculty Publications, Department of Child, Youth, and Family Studies by an authorized administrator of DigitalCommons@University of Nebraska - Lincoln. 
Authors

Robin S. Edelstein, Kristi Chin, Ekjyot K. Saini, Patty X. Kuo, Oliver C. Schultheiss, and Brenda L. Volling 


\title{
Adult attachment and testosterone reactivity: Fathers' avoidance predicts changes in testosterone during the strange situation procedure
}

\author{
Robin S. Edelstein, ${ }^{1}$ Kristi Chin, ${ }^{1}$ Ekjyot K. Saini, ${ }^{2}$ \\ Patty X. Kuo, ${ }^{3,5}$ Oliver C. Schultheiss, ${ }^{4}$ \\ and Brenda L. Volling ${ }^{1}$
}

\begin{abstract}
1 Department of Psychology, University of Michigan, Ann Arbor, MI, USA
2 Department of Human Development and Family Studies, Auburn University, Auburn, AL, USA

3 Department of Psychology, University of Notre Dame, Notre Dame, IN, USA

4 Department of Psychology, Friedrich-Alexander University, Erlangen, Germany

5 Current address: Child, Youth \& Family Studies, University of Nebraska-Lincoln,

Lincoln, NE, USA
\end{abstract}

Corresponding author - R.S. Edelstein, email redelste@umich.edu

\begin{abstract}
We assessed parents' testosterone reactivity to the Strange Situation Procedure (SSP), a moderately stressful parent-infant interaction task that pulls for parental nurturance and caregiving behavior. Parents (146 mothers, 154 fathers) interacted with their 1-year-old infants, and saliva samples were obtained pre- and post-task to assess changes in testosterone. We examined whether testosterone reactivity differed between mothers and fathers, the extent to which parents' characteristic approaches to closeness (i.e., adult attachment orientation) contributed to testosterone changes, and whether any influences of adult attachment orientation were independent of more general personality characteristics (i.e., the Big Five personality dimensions). Results revealed that mothers and fathers showed comparable declines
\end{abstract}

Published in Hormones and Behavior 112 (2019), pp 10-19.

doi 10.1016/j.yhbeh.2019.01.009

Copyright (c) 2019 Elsevier Inc. Used by permission.

Submitted 28 April 2018; revised 11 January 2019; accepted 16 January 2019;

published 28 March 2019. 
in testosterone during the SSP, and that these declines were attenuated among fathers with a more avoidant attachment orientation (i.e., those less comfortable with closeness). Associations between fathers' avoidance and testosterone reactivity were statistically independent of broader personality traits. Our findings provide some of the first evidence for short-term changes in both mothers' and fathers' testosterone in contexts that pull for nurturance. Moreover, these findings demonstrate that individual differences in adult attachment may play an important role in understanding such changes. We discuss possible explanations for gender differences in associations between adult attachment and parents' testosterone reactivity, and the extent to which testosterone reactivity might be sensitive to changes in context for mothers versus fathers.

Keywords: Testosterone, Parenting, Mothers, Fathers, Strange situation, Infants, Gender, Adult attachment, Avoidance, Personality

\section{Introduction}

Testosterone is a steroid hormone that has been linked with both aggression and nurturance in the context of close relationships (van Anders et al., 2011; Wingfield et al., 1990). Men with lower levels of baseline or endogenous testosterone, for instance, report more parental investment, greater empathy in response to infant cries, and less aggression toward romantic partners compared to their higher testosterone counterparts (Fleming et al., 2002; Kaiser and Powers, 2006; Mascaro et al., 2013; Soler et al., 2000). Fewer relevant studies have included women, but in women, lower testosterone has similarly been linked with more positive feelings toward children (Deady et al., 2006) and more pro-social tendencies such as nurturance and empathy (Harris et al., 1996).

Close relationship experiences that promote nurturance, such as partnering and parenting, can also lead to declines in testosterone over time. For instance, in both men and women, testosterone is typically lower among people in committed romantic relationships compared to single individuals and among parents versus non-parents (e.g., Barrett et al., 2013; Edelstein et al., 2011; van Anders and Goldey, 2010). Longitudinal research (primarily conducted among men) suggests that declines in testosterone are most pronounced among people who are more invested in these relationships (e.g., Gettler et al., 2011b; Saxbe et al., 2017). Taken together, these findings point to the potential role of nurturant experiences in changes in testosterone over time. 
Yet, there is considerable variability in people's endogenous testosterone levels and in their testosterone responses to close relationship experiences (e.g., Maestripieri et al., 2010; van Anders et al., 2014), and much less is known about the sources of this variability. Why might some people show larger changes in testosterone than others as a function of interactions with close others? In the current study, we examined a predictor of testosterone reactivity that should be particularly relevant in the context of close relationships: individual differences in adult attachment orientation, or people's characteristic approach to closeness and intimacy (Shaver and Mikulincer, 2007). We assessed changes in parents' testosterone following the Strange Situation Procedure (SSP), a parent-infant interaction task that is typically stressful for infants and thus likely to elicit parental nurturance or caregiving. We were particularly interested in the extent to which parents' attachment orientations were associated with testosterone reactivity and whether such associations were observed for both mothers and fathers. Additionally, given links between adult attachment orientation and more general dimensions of personality, we also assessed the extent to which individual differences in attachment contributed unique variance to testosterone reactivity above and beyond the "Big Five” personality factors (i.e., neuroticism, extraversion, agreeableness, openness to experience, conscientiousness, John et al., 2008). In the following paragraphs, we briefly describe relevant research on changes in testosterone as a function of nurturant interactions, as well as the rationale for expecting attachment-related differences in testosterone responses or reactivity to those interactions.

\subsection{Testosterone reactivity in close relationship contexts}

According to life history and evolutionary perspectives on parenting, there are tradeoffs with respect to the advantages of high versus low testosterone in mating versus parenting contexts (van Anders et al., 2011; Wingfield et al., 1990). That is, in situations that necessitate or foster competition for resources, sexual partners, or social status, higher levels of testosterone are thought to be particularly beneficial; in contrast, in situations that necessitate or foster nurturance or caregiving, lower levels of testosterone are thought to be particularly beneficial. Indeed, in both laboratory and field settings, testosterone 
tends to decrease following experiences that foster or encourage nurturance, whereas testosterone tends to increase following experiences that foster or encourage aggression, dominance or competition (see Edelstein and Chin, 2018; Zilioli and Bird, 2017, for review). For example, in one laboratory study, men showed decreases in testosterone after listening to recorded infant cries, and declines in testosterone were strongest among men who reported a desire to comfort the infant (Storey et al., 2000). van Anders et al. (2012) further demonstrated that men's testosterone responses to a crying infant doll depended on the quality of their interactions with that infant: Men who were given the opportunity to provide comfort to the doll tended to show decreases in testosterone following the interaction, but those who did not have this opportunity tended to show increases in testosterone, perhaps due to protective or defensive responses. Increases in testosterone might also have resulted from stress-related activation of the sympathetic nervous system and the ensuing stimulating effect of adrenaline on testosterone release (Sapolsky, 1987).

Flexibility in testosterone responses to nurturant situations may also be adaptive in producing optimal outcomes. For instance, in a recent study, fathers with lower baseline testosterone in the immediate postnatal period reported greater contributions to childcare compared to fathers with higher baseline testosterone levels (Kuo et al., 2018). Long-term declines in father's testosterone have also been linked with better parenting outcomes (Edelstein et al., 2017; Gettler et al., 2011b), suggesting that such changes may be beneficial or adaptive in promoting parental behavior. Much less is known about women's testosterone reactivity in caregiving situations and the long-term implications of such reactivity; however, in one recent study, women similarly showed pre- to post-interaction declines in testosterone after providing care to a crying infant doll (Voorthuis et al., in press).

Of note, significant pre- to post-interaction changes in testosterone have not generally been documented in studies in which fathers simply sat or played with their young children (e.g., Gettler et al., 2011a; Gray et al., 2007; Kuo et al., 2018; Storey et al., 2011). These findings suggest not only that the changes observed in previous research cannot be attributed simply to the passage of time, but also that situations that pull more strongly for parental caregiving behavior, such as the SSP, might be necessary to elicit short-term changes in men's 
testosterone. In fact, in a study of parents expecting their second child, Kuo et al. (2016) found that fathers showed significant declines in testosterone after participating in the SSP with their infants. Larger declines in testosterone, in turn, were associated with more positive parenting behavior during a subsequent parent-child interaction task. In the current report, we use data from this sample to assess predictors of testosterone reactivity during the SSP, which were not examined by Kuo et al. We also include assessments of mothers' as well as fathers' testosterone, to advance understanding of sex or gender differences in testosterone reactivity in nurturant contexts and the predictors of such reactivity.

Although much less is known about changes in women's versus men's testosterone as a function of caregiving, there are reasons to expect that mothers and fathers might show similar testosterone responses to the SSP. That is, despite relatively large sex difference in baseline testosterone levels, the magnitude of testosterone changes as a function of partnering and parenting are often similar for men and women (see Edelstein and Chin, 2018). For instance, as described earlier, in a recent laboratory study, female undergraduate students (none of whom had children) showed significant declines in salivary testosterone after providing care to a crying infant doll (Voorthuis et al., in press), much like those observed among male students by (van Anders et al., 2012). Unfortunately, very few studies that assess changes in testosterone during laboratory interactions with partners or children include both men and women. Moreover, the extent to which Voorthuis et al.'s findings might generalize to parents and their interactions with their own children, is not yet clear; however, such findings suggest that mothers as well as fathers might show declines in testosterone following the SSP.

1.2. Individual differences in adult attachment orientation as predictors of testosterone reactivity

Individual differences in adult attachment are generally conceptualized as a person's position on two conceptually independent dimensions: attachment-related avoidance and anxiety (Brennan et al., 1998). Attachment avoidance is characterized by discomfort with closeness and intimacy, and a compulsively "self-reliant" approach 
to relationships that emphasizes autonomy and independence over connectedness (Cassidy and Kobak, 1988; Wardecker et al., in press). Attachment anxiety is characterized by ambivalent feelings toward intimacy, concern about abandonment, and preoccupation with relationships and relationship partners (Campbell and Marshall, 2011; Mikulincer et al., 2002). People who report low levels of both avoidance and anxiety are considered to have a more secure (vs. insecure) attachment orientation.

Importantly, individual differences in attachment are thought to be most relevant and are most likely to be expressed in the context of close relationships and under conditions of stress or threat (e.g., Edelstein et al., 2004; Simpson and Rholes, 2012). Further, although adult attachment is more often assessed in the context of romantic versus parent-child relationships, there are reasons to expect that such assessments reflect broader feelings and beliefs about relationships more generally. For instance, avoidant adults report more ambivalence about becoming parents, and they derive less meaning and satisfaction from the experience of parenting (e.g., Rholes et al., 1997, 2006). There are fewer consistent links between attachment anxiety (vs. avoidance) and parenting, but maternal attachment anxiety has been linked with postpartum declines in marital satisfaction (Rholes et al., 2001; Simpson et al., 2003) and with more angry/intrusive parenting styles (Adam et al., 2004).

Additionally, although very few studies have assessed attachmentrelated differences in baseline testosterone levels or changes in testosterone, there are reasons to expect that attachment avoidance might be particularly relevant for understanding testosterone reactivity. Specifically, higher levels of testosterone have been linked with many correlates of avoidance, including poorer romantic relationship quality, a preference for short- versus long-term romantic relationships, and lower parental investment (Edelstein et al., 2011; Edelstein et al., 2014; Rholes et al., 2006; van Anders et al., 2007). Attachment avoidance has also been positively correlated with baseline testosterone levels among male undergraduate students (Sankar, 2015; Turan et al., 2014) and with markers of prenatal testosterone exposure (2D:4D digit ratio) in male and female youth (Del Giudice and Angeleri, 2016). We are not aware of studies linking attachment avoidance with testosterone changes or reactivity, but given that avoidant individuals may have higher baseline testosterone levels, and are generally less 
psychologically reactive toward others needing care (e.g., Edelstein et al., 2004), we expected that avoidant parents might also show smaller declines in testosterone as a function of participating in the SSP. To our knowledge, attachment-related anxiety has not been linked with baseline levels of testosterone or with testosterone reactivity. Thus, we assessed attachment- related anxiety, given its associations with attachment-related avoidance and with parenting (Edelstein et al., 2004), but we did not make predictions about associations between anxiety and testosterone reactivity.

Finally, we investigated the extent to which individual differences in attachment contributed unique variance to differences in testosterone reactivity above and beyond the contribution of more general personality traits. Attachment avoidance and anxiety are generally considered distinct from other broader measures of personality, such as the Big Five dimensions, but both attachment dimensions are typically positively correlated with neuroticism and negatively correlated with extraversion, agreeableness, and conscientiousness (e.g., Noftle and Shaver, 2006). These dimensions of personality have, in turn, been associated with parental behavior (e.g., parental sensitivity, Belsky et al., 1995) and (in some cases) baseline testosterone levels (Baucom et al., 1985; Sellers et al., 2007; Smeets-Janssen et al., 2015; but see Dabbs Jr. et al., 1990). Thus, there are reasons to expect that broad personality dimensions assessed by the Big Five might predict testosterone reactivity in the context of parent-child interactions. However, individual differences in adult attachment tend to be more influential than broad measures of personality in the context of close relationships (e.g., Edelstein et al., 2004; Noftle and Shaver, 2006), so we expected that parents' attachment orientation would contribute unique variance to their testosterone responses over and above any contributions of the Big Five personality dimensions.

\section{Method}

\subsection{Overview of study design and recruitment}

Participants were part of a larger longitudinal study of changes in family functioning after the birth of a second child (see Kuo et al., 2016; Volling et al., 2017, for additional details). Both mothers and 
fathers were assessed at five time points: prenatally (during the mother's third trimester of pregnancy), and at 1, 4, 8, and 12 months following the infant's birth. Women pregnant with their second child were recruited via advertisements and flyers posted in child care centers, local hospitals, pediatricians' offices, childbirth education classes, and obstetric clinics. Once families agreed to participate $(N=241$ out of 408 eligible), the first prenatal home visit was scheduled and the study was explained in greater detail with an opportunity for parents to ask questions prior to consenting. Families were compensated $\$ 300$ for completing all five time points.

Measures of parents' adult romantic attachment orientation were completed at the 12-month visit; measures of parents' personality (i.e., the Big Five personality dimensions) were completed during the prenatal session. At 12 months, two laboratory visits (one each for mothers and fathers, counterbalanced) were conducted to assess parent- infant attachment security using the Strange Situation Procedure (Ainsworth et al., 1978). Parents were also asked at 12 months to participate in a hormonal substudy that involved collecting saliva during the 12-month lab visits. During these visits, saliva samples were collected to assess changes in testosterone during the SSP. Each parent had the opportunity to opt in or out of this substudy independent of his or her partner, and the overall goals of the larger investigation. A total of 352 individuals (174 fathers) participated in the hormone substudy.

\subsection{Participants}

Participants in the current report include the 308 individuals (157 fathers) who completed the measure of adult romantic attachment, participated in the 12-month laboratory session, and had measures of both pre- and post-SSP salivary testosterone available. The majority of individuals (91\%) participated with their partner. An additional 12 people (7 fathers) participated in the laboratory session but did not complete the adult attachment measure, and 32 participants (10 fathers) had missing or unusable data for at least one of the testosterone assessments, due to errors in sample collection or assay (e.g., low sample volume, improperly secured collection tubes), and are not included in this report. 
Fathers in the current sample ranged in age from 24 to 46 years $(M=33.32 ; S D=4.51)$; mothers from 20 to 41 years $(M=31.99$; $S D=3.82$ ). Fathers' self-reported race/ethnicity was $87.9 \%$ European American, 3.8\% Black or African American, 3.2\% Asian or Asian American, 3.2\% Hispanic, and 1.9\% of other ethnicities. Mothers' selfreported race/ethnicity was 89.4\% European American, 4.0\% Black or African American, 3.3\% Asian or Asian American, 2.0\% Hispanic, and $1.3 \%$ of other ethnicities. The majority of both fathers and mothers had a least a college degree ( $83 \%$ and $84 \%$, respectively). Twentytwo percent of participants reported a household income of $\$ 20,000$ to $\$ 59,999,40 \%$ reported a household income of $\$ 60,000$ to $\$ 99,999$, and $38 \%$ reported a household income above $\$ 100,000$. Participants had been married for 5.86 years on average $(S D=2.71)$. Thirty mothers reported using some form of hormonal contraception (19\%; 2 mothers did not provide this information), and nine mothers reported being pregnant at the 12-month follow-up (6\%; 13 mothers did not provide this information).

The 157 participating father-infant dyads did not differ significantly from the recruited sample of fathers in terms of age, race/ethnicity, education level, years of marriage, or infant's gender; however, fathers who participated in the hormone substudy had significantly higher household incomes than fathers who did not participate, $\chi^{2}(3)$ $=17.60, p=.001$. The 151 participating mother-infant dyads did not differ significantly from the recruited sample in race/ethnicity, years of marriage, or infant's gender; however, mothers who participated in the hormone substudy had significantly higher household incomes than mothers who did not participate, $\chi^{2}(3)=15.64, p=.001$. Mothers who participated were also somewhat older, $t(239)=1.84, p=.07$, and had somewhat higher levels of education, $\chi^{2}(2)=5.42, p=.07$, than mothers who did not participate. (There were no mothers with less than a high school education.)

\subsection{Study protocol}

All procedures were reviewed and approved by the University of Michigan Institutional Review Board. The laboratory visits were completed when infants were 12 or 13 months of age and order of completion was counterbalanced across mothers and fathers. The two laboratory 
sessions were conducted approximately one month apart $(M=38.79$ days, $S D=11.09$, range $=13-84$ days), between the hours of 7:57 and 19:27 h. Given diurnal changes in testosterone levels (Schultheiss and Stanton, 2009), we attempted to minimize time variation by scheduling visits between 13:00 and 18:00 h (34\% of visits); however, we prioritized families' scheduling availability due to the large number of visits ( $\sim 400$ across mothers and fathers) that needed to be coordinated, as well as the infants' feeding and nap schedules. We also included time of visit as a covariate in our analyses.

Upon arriving at the laboratory, a trained researcher explained the procedures for the hormone substudy. First, prior to the SSP, parents provided a baseline saliva sample while in the waiting room with their infants (who were being held, playing on the floor with toys, etc.). Second, parents participated with their infants in the SSP, which was videotaped for subsequent coding. Third, following the SSP, parents provided a second saliva sample (15-20 min after the first SSP separation). Finally, parents engaged in a second interaction task, in which they were asked to teach their infant how to play with a variety of toys, which was followed by the collection of a third and final saliva sample. This second task and third saliva sample are not considered here, given our focus on changes in testosterone as a function of the SSP and the fact that fathers did not show significant changes in testosterone between the second and third samples (see Kuo et al., 2016, for additional details).

\subsection{The strange situation procedure (SSP)}

The SSP (Ainsworth et al., 1978) assesses the quality of infant- parent attachment bonds through a series of separations, followed by reunions, that become increasingly stressful over the course of seven 3-minute episodes (following a 1-minute introduction to the room). Infants typically become visibly upset during the SSP; they often search for their parents during the separations and seek comfort and contact with the parent upon reunion. During the separations, parents were allowed to observe their infants through a one-way observation window, and could curtail the separation at their request, before returning to comfort their children during the reunion. As is standard practice when conducting the SSP, experimenters also curtailed separation episodes if they judged that the infant's distress had reached extreme levels. 


\subsection{Adult attachment}

Parents' romantic attachment orientation was assessed with the Adult Attachment Questionnaire (AAQ; Simpson et al., 1996). The 8- item avoidance subscale $\left(\alpha_{\text {mothers }}=0.83, \alpha_{\text {fathers }}=0.83\right)$ reflects an individual's discomfort with intimacy. A sample item for avoidance is, "I don't like people getting too close to me." The 9-item anxiety subscale $\left(\alpha_{\text {mothers }}=0.81, \alpha_{\text {fathers }}=0.76\right)$ reflects an individual's fear of abandonment. A sample item for anxiety is, "Others are often reluctant to get as close as I would like." Parents rated the extent to which they agreed with statements on both subscales, using a 7-point scale, ranging from 1 (strongly disagree) to 7 (strongly agree). Participants responded to this measure based on their current thoughts and feelings in intimate relationships.

\subsection{Parental personality}

Parents' personality was assessed with the Revised NEO Personality Inventory (NEO PI-R; McCrae and John, 1992). Participants rated the extent to which they agreed with each of 60 statements on a 5-point scale, ranging from 1 (strongly disagree) to 5 (strongly agree). Scores are then derived for five general dimensions of personality: neuroticism (sample item: "I often feel tense and jittery"; $\alpha_{\text {mothers }}=0.83$, $\alpha_{\text {fathers }}=0.80$ ), extraversion (sample item: "I really enjoy talking to people"; $\alpha_{\text {mothers }}=0.77, \alpha_{\text {fathers }}=0.73$ ), openness to experience (sample item: "I have a lot of intellectual curiosity"; $\alpha_{\text {mothers }}=0.79$, $\alpha_{\text {fathers }}=0.76$ ), agreeableness (sample item: "I generally try to be thoughtful and considerate"; $\alpha_{\text {mothers }}=0.78, \alpha_{\text {fathers }}=0.74$ ), and conscientiousness (sample item: "I am a productive person who always gets the job done"; $\alpha=0.81$ ).

\subsection{Salivary testosterone: collection and assessment}

Salivary testosterone assays are well-established, validated, and widely used in biobehavioral research with both men and women (Schultheiss and Stanton, 2009; van Anders et al., 2014). Salivary testosterone also correlates highly with free and total serum testosterone, although salivary measures are more sensitive to collection and storage artifacts compared to serum measures (Granger et al., 2004; 
Khan- Dawood et al., 1984; Magrini et al., 1986). Numerous studies document links between women's salivary testosterone and psychologically and/ or behaviorally meaningful outcomes (e.g., Edelstein et al., 2014; Endendijk et al., 2016; van Anders et al., 2009); however, some have argued that salivary measures may underestimate the strength of testosterone- behavior associations in women (Granger et al., 2004; Shirtcliff et al., 2002).

Saliva samples were collected in $50 \mathrm{ml}$ polypropylene tubes (United Lab Plastics) and participants provided $10 \mathrm{ml}$ of saliva per sample. The first sample served as a baseline after parents arrived in the laboratory (T1) and the second sample was taken 15-20 min after the first separation of the SSP, approximately 30-35 min after the first sample (T2). (As described above, a third saliva sample was collected but is not considered given our interest in changes pre- to post-SSP.) Participants were told to refrain from eating or drinking anything but water for at least 30 min before the laboratory visit. Saliva collection was stimulated via chewing sugar-free Trident Original gum. Although some brands and flavors of gum can affect testosterone results, this particular kind of gum has been shown to have minimal effects on testosterone results compared to other kinds of gum using similar assays (Dabbs Jr., 1991; Granger et al., 2004).

All samples were frozen at $-20{ }^{\circ} \mathrm{C}$ until assayed. Samples were analyzed by radioimmunoassay using a commercial kit from Siemens Healthcare that was modified for use with saliva according to published protocol (Campbell et al., 1999). Water-based dilutions of all standards and controls were prepared to determine salivary testosterone concentrations. Samples were assayed in duplicate and the mean levels for each sample were utilized for analysis. Controls were used to assess assay reliability. Samples from the same participants were processed in the same assay. The intra-assay coefficient of variation (CV) was $10.17 \%$ for fathers and $15.25 \%$ for mothers; the inter-assay CV was $21.22 \%$ for fathers and $20.71 \%$ for mothers. Our intra-assay CVs, and particularly our inter-assay CVs, are somewhat high, although similar values have been obtained in other samples that have assessed salivary testosterone in both men and women, including studies that find associations between women's testosterone and other outcomes (e.g., Edelstein et al., 2014; Liening et al., 2010; Raisanen et al., 2018). Higher CVs suggest greater measurement error in our testosterone estimates; however, it is worth noting that such error does not appear 
to differ notably by gender in our sample, suggesting that any gender differences in our findings are not clearly attributable to gender differences in the precision of our hormone assays. It is also worth noting that, for both men and women, the range of testosterone values that we report are very similar to those reported for samples of similarly aged participants (e.g., Keevil et al., 2017).

To examine changes in testosterone as a function of the SSP, we computed percent change scores (i.e., ((T2-T1) / T1)×100), a commonly used method of assessing short-term testosterone reactivity (e.g., Fleming et al., 2002; van Anders et al., 2012; Weisman et al., 2014). Unlike raw difference scores, percent change scores account for baseline differences in hormone levels and are thus generally preferred to difference scores, which can be difficult to interpret when there are large individual and/or gender differences in baseline hormone levels (e.g., van Anders et al., 2009). However, we also re-conducted all analyses using residualized change scores (i.e., predicting Time 2 testosterone levels from Time 1 testosterone levels and saving the unstandardized residuals) and using Time 1 testosterone scores as a covariate. All results presented below were virtually identical when these alternative metrics were used.

\subsection{Statistical analyses}

The Statistical Package for the Social Sciences (SPSS, version 24) was used to conduct all analyses. Mean differences between parents were assessed using paired samples $t$-tests (two-tailed) and associations were assessed using correlations. For multivariate analyses, we used dyadic models that account for the interdependence between parents using SPSS Mixed (Kenny et al., 2006) and we report unstandardized beta coefficients for these analyses.

As is standard practice in testosterone research (e.g., Carre et al., 2014; van Anders et al., 2012), data for four participants (one father) with testosterone levels $>3$ standard deviations above the mean for their gender at one or both time points were excluded from further analyses. An additional four participants (two fathers) had testosterone percent change values larger than three standard deviations above the mean for their gender and were also excluded from further analyses. Thus, subsequent analyses were conducted with 300 participants (154 fathers). 


\section{Results}

\subsection{Preliminary analyses}

Descriptive statistics and correlations among the primary study variables are presented by gender in Table 1, with values for mothers presented above the diagonal and values for fathers presented below the diagonal. As shown in Table 1, for both mothers and fathers, testosterone values at Time 1 were highly correlated with those at Time 2, indicating significant rank-order stability in testosterone levels from before to after the SSP. As expected, fathers had significantly higher testosterone levels than mothers at both Time 1, $t(132)=27.93, p<$ $.01, d=2.42$, and Time $2, t(132)=26.33, p<.01, d=2.28$. On average, both mothers and fathers showed declines in testosterone from pre- to post-SSP, and there were no significant gender differences in the extent of testosterone change, $t(132)=1.23, p=.22, d=0.11$. Attachment avoidance and anxiety also did not significantly differ by gender, $p$ 's $>$ 0.28. (Analyses based on independent samples t-tests, which include an additional 34 parents whose partners did not participate in the hormonal substudy, yielded virtually identical results.)

Also as shown in Table 1, attachment avoidance and anxiety were significantly positively intercorrelated for mothers but not for fathers. Neither avoidance nor anxiety were significantly correlated with mothers' or fathers' testosterone levels at either Time 1 or Time 2; however, more avoidant fathers showed smaller decreases in testosterone from

Table 1. Descriptive statistics and correlations among primary study variables.

\begin{tabular}{lllllllll} 
& 1 & 2 & 3 & 4 & 5 & $M$ & $S D$ & Range \\
\hline Baseline T (pg/ml; Time 1) & $\mathbf{0 . 1 4}$ & $0.81^{* *}$ & $-0.20^{*}$ & -0.03 & -0.03 & 15.42 & 6.60 & $1.10-35.68$ \\
Post-SSP T (pg/ml; Time 2) & $0.79^{* *}$ & $\mathbf{0 . 1 9 *}$ & $0.33^{* *}$ & -0.09 & 0.02 & 12.92 & 6.42 & $1.47-28.88$ \\
T1 - T2 Reactivity (\% change) & $-0.25^{* *}$ & $0.35^{*}$ & $\mathbf{0 . 0 8}$ & -0.10 & 0.06 & -13.62 & 29.63 & $-76.97-83.90$ \\
Attachment avoidance & 0.01 & 0.11 & $0.20^{*}$ & $\mathbf{0 . 1 4}$ & $0.45^{* *}$ & 3.11 & 0.95 & $1-5.88$ \\
Attachment anxiety & -0.03 & 0.03 & 0.09 & 0.15 & $\mathbf{0 . 2 2}$ & 2.52 & 0.95 & $1-6.00$ \\
$M$ & 66.11 & 59.00 & -9.25 & 3.22 & 2.41 & & & \\
$S D$ & 20.78 & 19.91 & 18.96 & 0.96 & 0.81 & & & \\
Range & $18.65^{-124.70}$ & $20.37-133.74$ & $-60.28-35.63$ & $1-6.13$ & $1-5.38$ & & & \\
\hline
\end{tabular}

Note. Descriptive statistics and correlations are presented above the diagonal for mothers $(N=146)$ and below the diagonal for fathers $(N=154)$; bolded values on the diagonal are within-couple correlations for couples in which both parents participated $(N=266)$.

${ }^{*} p<.05$

${ }^{* *} p<.01$ 
Time 1 to Time 2. Also, as shown on the diagonal, attachment anxiety and post-SSP testosterone levels were significantly positively intercorrelated within couples.

Correlations between the Big Five personality dimensions and key study variables are presented in Table $\mathbf{2}$. As shown in Table 2, for both mothers and fathers, there were no significant correlations between any of the Big Five dimensions and any of the testosterone variables; however, as expected, among both mothers and fathers, attachment avoidance was positively correlated with neuroticism and negatively correlated with agreeableness and extraversion. Attachment anxiety was also positively correlated with neuroticism and negatively correlated with agreeableness among both mothers and fathers. Additionally, attachment anxiety was negatively correlated with conscientiousness and extraversion among mothers. The Big Five dimensions were also moderately intercorrelated with one another (not shown), with correlations ranging from -0.51 to 0.35 . None of the within-dyad correlations were statistically significant, with the exception that couples tended to have similar levels of openness to experience, $r=0.30, p<.01$.

Table 2. Descriptive statistics and correlations between key variables and big five personality dimensions.

\begin{tabular}{|c|c|c|c|c|c|}
\hline & $\mathrm{N}$ & $\mathrm{E}$ & $\mathrm{O}$ & A & $\mathrm{C}$ \\
\hline \multicolumn{6}{|l|}{ Mothers $(N=146)$} \\
\hline Baseline T (pg/ml; Time 1) & $-0.03^{*}$ & -0.04 & -0.01 & -0.04 & 0.07 \\
\hline Post-SSP T (pg/ml; Time 2) & -0.04 & 0.00 & -0.07 & -0.03 & 0.02 \\
\hline $\mathrm{T} 1$ - T2 reactivity (\% change) & -0.05 & 0.03 & -0.12 & 0.01 & -0.05 \\
\hline Attachment avoidance & $0.47^{* *}$ & $-0.45^{* *}$ & 0.10 & $-0.44^{* *}$ & -0.12 \\
\hline Attachment anxiety & $0.58^{* *}$ & $-0.27^{* *}$ & 0.00 & $-0.46^{* *}$ & $-0.27^{* *}$ \\
\hline$M$ & 31.17 & 40.45 & 39.79 & 46.44 & $45 \cdot 51$ \\
\hline$S D$ & 7.89 & 6.37 & 6.88 & $5 \cdot 53$ & 7.07 \\
\hline \multicolumn{6}{|l|}{ Fathers $(N=154)$} \\
\hline Baseline T (pg/ml; Time 1) & -0.02 & 0.09 & 0.10 & -0.03 & 0.01 \\
\hline Post-SSP T (pg/ml; Time 2) & 0.04 & 0.07 & 0.03 & -0.04 & 0.05 \\
\hline $\mathrm{T} 1$ - T2 reactivity (\% Change) & 0.11 & -0.03 & -0.09 & -0.01 & 0.08 \\
\hline Attachment avoidance & $0.35^{* *}$ & $-0.48^{* *}$ & -0.01 & $-0.32 * *$ & 0.02 \\
\hline Attachment anxiety & $0.32^{* *}$ & 0.00 & 0.02 & $-0.25^{* *}$ & -0.06 \\
\hline$M$ & 27.81 & 40.35 & 40.79 & 44.62 & $44 \cdot 55$ \\
\hline$S D$ & $7 \cdot 38$ & 6.34 & 6.47 & $5 \cdot 53$ & 6.89 \\
\hline
\end{tabular}

Note. N: neuroticism; E: extraversion, O: openness to experience, A: agreeableness, C: conscientiousness.

$* p<.05$

${ }^{* *} p<.01$ 
We additionally examined several potential covariates-parents' age, time since last brushing teeth, time of day, body mass index (BMI), infant sex, seasonality. None of these variables were significantly included in subsequent analyses. For women, we also examined oral contraceptive use and pregnancy status as potential covariates, as these variables are often associated with baseline testosterone levels (e.g., van Anders et al., 2014). In the current study, women on oral contraceptives had lower testosterone levels at both time points, ts $(142)=-3.36$ and $-3.64, d s=-0.71$ and -0.77 , respectively, $p s<$ 0.01, but testosterone levels did not differ by pregnancy status, $p s>$ o.21. Moreover, neither was associated with or moderated associations with testosterone reactivity in subsequent analyses, so these variables were not considered further. Excluding pregnant women and those using hormonal contraceptives yielded a similar pattern of findings to those reported here.

\subsection{Multilevel models predicting testosterone reactivity}

We next conducted multilevel modeling analyses to examine: (1) the independent contributions of each attachment dimension to testosterone reactivity while controlling for time of day, (2) whether these associations differed by gender, and (3) whether any associations between parents' attachment and testosterone reactivity remained significant after accounting for broader personality constructs as assessed by the Big Five. We used dyadic data analyses that account for the interdependence between couple members (Kenny et al., 2006); these models also allow for missing data, such that estimates are still provided for participants whose partners did not participate in the hormone substudy. Time of day, gender, attachment avoidance, attachment anxiety, and the two-way interactions between gender and (1) the attachment dimensions and (2) time of day were included as predictors of testosterone reactivity. All continuous variables were mean-centered, and gender was contrast coded, such that mothers = 1 and fathers $=-1$.

Results from this analysis, shown in Table 3, revealed a significant interaction between attachment avoidance and gender. Decomposing this interaction indicated that, consistent with the zero-order correlations described earlier, avoidant fathers showed smaller declines in testosterone reactivity as a function of the SSP, $b=3.87, S E=1.56$, 
Table 3. Multilevel model predicting testosterone reactivity to the strange situation procedure.

\begin{tabular}{|c|c|c|c|}
\hline & $b$ & $S E(B)$ & $t$ \\
\hline Intercept & -12.04 & 1.49 & $-8.06 * *$ \\
\hline Time of day & 0.58 & 0.48 & 1.20 \\
\hline Gender & 2.13 & 1.42 & 1.50 \\
\hline Attachment avoidance & -0.62 & 1.65 & $-0.38 *$ \\
\hline Attachment anxiety & 2.61 & 1.73 & 1.51 \\
\hline Gender $\times$ time of day & 0.49 & 0.47 & 1.04 \\
\hline Gender $\times$ avoidance & $4 \cdot 49$ & 1.64 & $2.74^{* *}$ \\
\hline Gender×anxiety & -1.62 & 1.72 & -0.94 \\
\hline
\end{tabular}

Note. $N=300$; Dependent variable is percent change in testosterone over baseline. Effects are reported as unstandardized regression coefficients from the final model including all main effect and interaction terms; Gender: $-1=$ women, $1=$ men.

${ }^{*} p<.05$

$* * p<.01$

$t(150.35)=2.48, p=.01$; the association between mothers' avoidance and testosterone reactivity was in the opposite direction and was not statistically significant, $b=-5.12, S E=2.89, t(146.76)=-1.77$, $p=.08$. Also consistent with the zero-order correlations, attachmentrelated anxiety was not significantly associated with changes in parents' testosterone. Results were unchanged when we included partner effects (e.g., fathers' avoidance predicting mothers' testosterone reactivity), and none of these partner effects were statistically significant, all ps > 0.13. Results were also virtually identical to those reported here when the two-way interaction between attachment avoidance and anxiety (and the three-way interaction with gender) were included.

Additionally, because infants might be more distressed when experiencing the SSP for the second versus first time, we included order of SSP participation (first versus second session) in the analyses presented above, for families in which both parents participated in the SSP. ${ }^{1}$ Parents' testosterone reactivity did not differ according to

1. Indeed, in our sample, infants' observer-rated distress was higher during the second compared to the first SSP, $b=-0.21, S E=0.05, t(145.09)=-4.13, p<.01$, and with mothers compared to fathers, $b=-0.10, S E=0.05, t(148.70)=-2.00, p<.05$; however, infant distress was unrelated to any other study variables, including parents' attachment orientation and their testosterone reactivity. Moreover, including infant distress in our models did not change or moderate any of our study findings. Thus, in the interest of parsimony, this variable is not included in subsequent analyses. 
whether they participated in the first versus second SSP with their infant, $b=0.58, S E=1.50, t(134.32)=0.38, p=.70$. The interaction between order of participation and gender was also nonsignificant, $b=-2.43, S E=1.56, t(135.54)=-1.56, p=.12$; however, there was a significant 3-way interaction between order of participation, gender, and attachment avoidance, $b=3.87, S E=1.71, t(210.84)=2.26$, $p=.03$. Decomposing this interaction revealed that, consistent with findings reported earlier, more avoidant fathers showed smaller declines in testosterone, $b=-4.14, S E=1.72, t(127.34)=2.40, p=.02$, and this effect was not moderated by session order, $b=1.25, S E=1.76$, $t(127.36)=0.71, p=.48$. For mothers, however, avoidance was associated with significantly larger declines in testosterone only if they participated in the first, $b=-11.35, S E=-4.40, t(65)=-2.58, p=.01$, but not the second session, $b=1.88, S E=3.90, t(65)=0.48, p=.63$.

We also assessed whether our effects might be driven by artifacts due to the timing of the laboratory assessments. We included the two- and three-way interactions among time of day, the attachment dimensions, and gender in our multilevel model. The avoidance by gender interaction remained statistically significant in this analysis, $b=4.23, S E=1.61, t(218.73)=2.62, p<.01$, and the association between fathers' avoidance and testosterone reactivity remained positive and statistically significant, $b=3.92, S E=1.56, t(149.72)=2.50$, $p=$.01. The 3-way interaction among avoidance, gender, and time of day was statistically significant, however, $b=-1.26, S E=0.52$, $t(195.61)=-2.44, p=.02$. Decomposing this interaction revealed that it was driven by a significant two-way interaction between avoidance and time of day for mothers, $b=2.50, S E=0.94, t(141.90)=2.66$, $p<.01$, such that the negative association between mothers' avoidance and testosterone reactivity (described earlier) was statistically significant only earlier versus later in the day. Thus, our findings do not appear to be confounded by time of day artifacts. (There were also no significant zero-order correlations between the attachment dimensions and session time for either mothers or fathers, $p s>0.14$.) We also reconducted all analyses using residualized percent changes in testosterone scores that controlled for time of day (rather than including time of day as a variable in our analyses). All results were virtually identical, again suggesting that our results were not driven by artifacts due to session timing. 
Finally, to test whether parents' attachment orientation predicted testosterone reactivity above and beyond the contribution of more general personality characteristics, we included the Big Five personality dimensions in the multilevel model described above, along with the five two-way interactions between gender and each personality dimension. None of these additional predictors were statistically significant, $p$ 's $>0.11$. Further, the gender by avoidance interaction remained statistically significant with the Big Five dimensions in the model, $b=3.90, S E=1.90, t(223.10)=2.05, p=.04$. Thus, the associations between parents' attachment avoidance and testosterone reactivity were statistically independent of the contribution of the Big Five personality dimensions. ${ }^{2}$

2. Other published reports from this dataset have linked components of parental behavior during the subsequent teaching task with mothers' and fathers' adult attachment orientations (in combination with parents' implicit affiliation and power motives, Safyer et al., in press) and with fathers' testosterone reactivity during the SSP (Kuo et al., 2016). In the interest of completeness and transparency, we conducted additional analyses to examine whether parents' testosterone reactivity during the SSP served as a mediator between parental attachment and positive parental behavior-a composite of parental sensitivity, positive regard, simulation of cognitive development, and (reverse-scored) intrusiveness, negative regard, and detachment-as measured and reported in Kuo et al. That is, can the association between parental attachment and positive parenting behavior during the teaching task be explained by parents' testosterone reactivity during the preceding SSP? In dyadic analyses, we first examined whether parents' attachment was associated with parental behavior during the teaching task: Attachment avoidance was unrelated to positive parenting behavior among both mothers, $b=-0.39, p=.20$, and fathers, $b=0.13, p=.65$. Attachment anxiety was also unrelated to positive parenting behavior among mothers, $b=0.23, p=.46$, but was negatively related to positive parenting among fathers, $b=-1.06, p<.01$. That is, more anxious fathers behaved less positively toward their children during the teaching task. When testosterone reactivity was included in the aforementioned model, to test the proposed mediation of parental attachment and behavior by testosterone reactivity, the association between fathers' anxiety and positive parenting behavior remained significant, suggesting that this association was not explained by testosterone reactivity. Moreover, fathers' testosterone reactivity during the SSP was negatively associated with positive parenting behavior during the teaching task, $b=-.03, p=.03$, as has been reported previously by Kuo et al. (2016), suggesting that this link was not accounted for by parental attachment. Mothers' testosterone reactivity was not associated with subsequent behavior, $b=-.01$, $p=.42$. Given that parenting behavior during the subsequent teaching task was not part of our a priori framework or hypotheses, we do not consider this variable further here, but readers may consult Kuo et al. (2016) and Safyer et al. (in press) for additional details about and analyses of parental behavior from this dataset. 


\section{Discussion}

In the current study, we assessed predictors of parents' testosterone reactivity during the SSP, a stressful situation that is likely to elicit parental care and responsiveness. We were especially interested in the extent to which individual differences in adult attachment contributed to parents' testosterone changes; whether associations between attachment and testosterone reactivity differed between mothers and fathers; and whether any influences of adult attachment were independent of more general personality characteristics. Previous research provides evidence for short-term declines in testosterone following nurturant interactions, such as providing care to a crying infant (Kuo et al., 2016; van Anders et al., 2012); however, the vast majority of this research has been conducted with men and/or fathers, leaving open many questions about changes in women's testosterone during nurturant interactions. Moreover, relatively little is known about individual differences in testosterone reactivity, that is, why some people may show smaller or larger testosterone changes as a function of nurturant interactions.

Our findings demonstrated, first, that mothers and fathers showed comparable declines in testosterone after participating in the SSP. To our knowledge, only one study has examined changes in women's testosterone during such interactions (Voorthuis et al., in press). Voorthuis et al. asked undergraduate students (none of whom had children) to provide care to a crying infant doll, and their salivary testosterone was assessed pre- and post-interaction. On average, women showed significant declines in testosterone after providing care to the doll. That women were not interacting with their own children, or even a real infant, suggests that changes in testosterone may be a function of nurturance more generally as opposed to caring for one's own child specifically. Of course, changes in testosterone could be larger when one interacts with one's own versus another (real or simulated) child; this would be an interesting comparison for future research. Nevertheless, and perhaps more importantly, our findings provide evidence for similar patterns of testosterone change across gender in the context of parent-child interactions.

It is important to note that changes in testosterone have generally not been observed when parents simply sit quietly with their children or perform other non-nurturant tasks (e.g., Gettler et al., 2011a). In 
fact, in our own sample, fathers showed declines in testosterone preto post- SSP but not following a subsequent teaching task (Kuo et al., 2016). Thus, the post-SSP declines we observed are unlikely due simply to the passage of time; however, ideally, future studies could compare different kinds of interactions within the same study to allow for stronger inferences about the effects of nurturance per se. Further, one limitation of our study design is that all dyads completed the SSP prior to the teaching task, which makes it impossible to know how task order may have influenced hormone reactivity. The tasks were ordered in this way to maintain the consistency of the SSP as a measure of parent-infant attachment, and because the teaching task gave dyads an opportunity to have a more positive, and less stressful, interaction before leaving the lab. However, we cannot rule out the possibility that parents might have shown changes in testosterone following the teaching task if they had participated in that task at the beginning of the laboratory session.

To our knowledge, our study is the first to assess both mothers' and fathers' testosterone reactivity to the same nurturance task in the same study. One advantage of this approach is that mothers and fathers interacted with the same infant, thus holding at least some aspects of this interaction constant. One disadvantage of this approach is that couples' attachment orientations and physiology may not necessarily be independent, and in fact our preliminary analyses suggested some concordance between couple members' attachment anxiety and testosterone levels. We did not find that parents' hormone changes were significantly intercorrelated, however, including in dyadic analyses that accounted for the interdependence between couple members. These findings suggest that parents' responses to the SSP may have been fairly independent, at least in terms of testosterone reactivity.

Our findings also extend prior research on parents' testosterone reactivity by examining predictors of testosterone changes during the SSP. We specifically focused on individual differences in adult attachment, which have been associated with parental behavior in stressful or nurturance-eliciting situations and with baseline hormone levels. Specifically, higher levels of attachment avoidance, or discomfort with closeness, have been linked with less responsive caregiving in stressful contexts (e.g., Edelstein et al., 2004) and with higher levels of baseline testosterone (e.g., Turan et al., 2014). These findings led 
us to predict that more avoidant parents would show smaller declines in testosterone following the SSP. This hypothesis was confirmed for fathers but not for mothers: Fathers who reported higher levels of attachment avoidance showed smaller pre- to post-SSP declines in testosterone. Among mothers, attachment avoidance was, if anything, associated with larger declines in testosterone, although these associations were statistically significant only when infants participated in the SSP with mothers before fathers or earlier in the day. Attachment anxiety, or concern about abandonment, was not significantly associated with testosterone reactivity among either mothers or fathers.

Why might avoidance be more strongly associated with testosterone reactivity in fathers versus mothers? Perhaps the SSP is a more novel scenario for fathers than for mothers, in that mothers are more often in the role of primary caretaker and are more often socialized to play caregiving roles (Eagly and Wood, 1999). Indeed, in our sample, couples reported that mothers spent more time on childcare relative to fathers (Kuo et al., 2016). ${ }^{3}$ Thus, the SSP may have been less stressful for avoidant mothers than for avoidant fathers, and therefore, less likely to activate avoidant defensive behaviors, which could ultimately influence testosterone reactivity. That mothers' avoidance predicted testosterone reactivity only when infants participated in the SSP for the first time further suggests that something about the novelty of the situation may have contributed to these effects. Unfortunately, we did not measure parents' distress or emotional responses during this procedure, but future research could test this possibility by examining parents' self-reported or behavioral expressions of distress during the SSP. It is also worth noting that parents' attachment anxiety and avoidance were unrelated to infants' observer-rated distress during the SSP, suggesting that children did not respond differently to the SSP as a function of their parents' attachment orientations. Moreover, as discussed earlier, on

3. Our measure of division of infant care was based on a joint task in which parents were asked to come to an agreement about the proportion of infant care done by each parent. Mothers and fathers in our study agreed that mothers did more infant care than fathers; however, division of infant care was not significantly associated with parents' attachment or their testosterone reactivity during the SSP. Further, when division of infant care was included in our analyses, it was not a statistically significant predictor of testosterone reactivity, and did not moderate any of our findings. It is worth noting, however, that this measure does not strictly assess the amount of time that parents spend with their children, but rather the relative proportions of child care engaged in by each parent. 
average, mothers and fathers showed comparable declines in testosterone following the SSP, so it is unlikely that mothers on the whole responded differently than fathers to this procedure (at least with respect to this neuroendocrine measure).

Future research might also assess parents' observable behavior during the SSP or other kinds of stressors to examine whether more avoidant mothers and fathers differ in how they interact with their children and the extent to which such differences contribute to the differences in testosterone reactivity that we observed here. As noted earlier, van Anders et al. (2012) found that (male) participants who had the opportunity to comfort a crying doll showed declines in testosterone following their interaction with the doll; however, participants who could not comfort the doll showed increases in testosterone. Perhaps more avoidant fathers felt less confident in their abilities to comfort their infants, in general, and during the SSP, potentially leading to smaller declines in testosterone as a function of this interaction.

Recent findings in fact suggest that individual differences in testosterone and testosterone variability may be differentially correlated with parental behavior for mothers versus fathers: Endendijk et al. (2016) examined diurnal changes in mothers' and fathers' testosterone in relation to parental behavior during a play task with their young children. For fathers, greater testosterone variability (i.e., sharper declines in testosterone from the morning to the evening assessment) was associated with greater sensitivity toward children and greater respect for their autonomy during the play task. Findings for mothers were in the opposite direction: greater testosterone variability was associated with less sensitivity and less respect for children's autonomy. Moreover, among mothers, higher evening testosterone levels predicted more sensitive parenting during the play session. Endendijk et al. (2016) speculate that these differences might reflect the different evolutionary tradeoffs between parenting and mating/competition that may be salient for mothers versus fathers, such that fathers may benefit more from flexibility in testosterone responses when interacting with young children (versus, for instance, competing for resources at work). Further research is needed to understand the extent to which these differences might contribute to individual differences in testosterone reactivity during naturalistic stressors such as the SSP, and particularly whether they may impact the experiences of avoidant mothers versus fathers. 
It is also important to note, however, that measurement issues may have contributed to the gender differences that we observed here. As is typical of salivary testosterone research, in our study women's testosterone was more restricted in range compared to men's. Moreover, salivary assay measurement error is typically larger for the lower (female) range of testosterone measurements than for the higher (male) range. And finally, although we used a chewing gum to stimulate saliva flow that has not interfered with testosterone measurement in past research (Dabbs Jr., 1991), its use has not been validated specifically for the assay we employed in our study. These factors can increase measurement error in women's compared to men's salivary testosterone, which may have made it more difficult for us to observe associations between testosterone and other variables in mothers versus fathers.

Further research that includes other kinds of nurturant tasks and/ or other neuroendocrine markers might also help to shed light on these gender differences. For instance, there is some evidence that avoidant women show smaller increases in estradiol (a steroid hormone associated with bonding and caregiving) after viewing emotionally intimate stimuli (Edelstein et al., 2012). Perhaps other hormones, such as estradiol, are more closely linked with women's attachment avoidance in nurturant contexts. It is also possible that more intimate tasks, such as emotional parent-child conversations, would be more likely to elicit changes in avoidant women's hormones. Research on the dual-hormone hypothesis further suggests that associations between testosterone and behavior might be most pronounced or observable when baseline levels of cortisol are relatively low (see Mehta and Prasad, 2015). In the current study, therefore, it is possible that changes in testosterone might have been dampened among parents who experienced increases in cortisol during the SSP. Moreover, insofar as cortisol changes are linked with individual differences in attachment and/or gender, such changes could have contributed to the gender differences we observed here (e.g., Beck et al., 2013). Future studies might therefore include more diverse assessments of neuroendocrine reactivity and observations of mothers and fathers in different nurturant and emotional contexts.

Our findings additionally demonstrated that attachment avoidance predicted testosterone reactivity independently of any associations between these variables and more general personality dimensions as 
assessed by the Big Five. Broad personality traits, such as extraversion and agreeableness, have been linked with individual differences in adult attachment and parental behavior (e.g., Belsky et al., 1995), and in some cases with baseline testosterone levels (e.g., Baucom et al., 1985), suggesting that it may be important to account for these variables in analyses of parents' testosterone reactivity. In the current study, however, none of the Big Five personality dimensions were significantly correlated with baseline testosterone levels or with testosterone reactivity among either mothers or fathers. Moreover, although these personality variables were associated with avoidance and anxiety in expected ways, our main findings were unchanged when the personality variables were included in regression analyses. Thus, at least in the current study, we found very little evidence for links between parents' testosterone and more general personality characteristics.

Of course, our findings should be interpreted in the context of the unique characteristics of our sample. For instance, all of the children in our sample had older siblings. To the extent that second-time parents are older and more experienced, more confident in their parenting skills, and/or limited in the time they have to spend with any individual child compared to first-time parents, our findings may not extend to first-time parents. Further, our findings may also not generalize to or be replicated using younger, college-age, non-parent populations interacting with simulated infant dolls, and this is an areas worthy of further investigation. The families in our sample were also married heterosexual couples, largely of European American descent, and generally of relatively high socio-economic status (as indexed by average levels of education and income), so it will be important for future research to assess the extent to which our findings generalize to other demographic groups.

Despite these limitations, our findings demonstrated that mothers and fathers showed similar patterns of testosterone reactivity during the SSP, a stressful parent-child interaction that pulls for nurturance (Kuo et al., 2016). We additionally shed light on a potentially important predictor of such changes, particularly for fathers, in that more avoidant fathers showed smaller declines in testosterone during the SSP. These associations were independent of more general personality characteristics, suggesting that individual differences in adult 
attachment orientations make a unique contribution to understanding parents' hormone changes. Future research might benefit from focusing on other kinds of nurturant interactions, additional hormones, and more diverse samples of parents.

Acknowledgments - This research was supported by grants (Ro1HDo42607, Ko2HDo47423) from the Eunice Kennedy Shriver National Institute of Child Health and Human Development to Brenda L. Volling, and a faculty grant to Volling and Schultheiss from the Office of Research at the University of Michigan. We are grateful to the parents of the Family Transitions Study and to the research assistants who provided invaluable help with data collection.

\section{References}

Adam, E.K., Gunnar, M.R., Tanaka, A., 2004. Adult attachment, parent emotion, and observed parenting behavior: mediator and moderator models. Child Dev. $75,110-122$.

Ainsworth, M.D.S., Blehar, M.C., Waters, E., Wall, S., 1978. Patterns of Attachment: A Psychological Study of the Strange Situation. Lawrence Erlbaum Associates, Hillsdale, NJ.

van Anders, S.M., Tolman, R.M., Volling, B.L., 2012. Baby cries and nurturance affect testosterone in men. Horm. Behav. 61, 31-36.

van Anders, S.M., Goldey, K.L., Bell, S.N., 2014. Measurement of testosterone in human sexuality research: methodological considerations. Arch. Sex. Behav. 43, 231-250.

Barrett, E. S., et al., 2013. Marriage and motherhood are associated with lower testosterone concentrations in women. Horm. Behav. 63, 72-79.

Baucom, D.H., Besch, P.K., Callahan, S., 1985. Relation between testosterone concentration, sex role identity, and personality among females. J. Pers. Soc. Psychol. 48, 1218-1226.

Beck, L.A., Pietromonaco, P.R., DeBuse, C.J., Powers, S.I., Sayer, A.G., 2013. Spouses' attachment pairings predict neuroendocrine, behavioral, and psychological responses to marital conflict. J. Pers. Soc. Psychol. 105, 388-424.

Belsky, J., Crnic, K., Woodworth, S., 1995. Personality and parenting: exploring the mediating role of transient mood and daily hassles. J. Pers. 63, 905-929.

Brennan, K.A., Clark, C.L., Shaver, P.R., 1998. Self-report measurement of adult attachment: an integrative overview. In: Simpson, J.A., Rholes, W.S. (Eds.), Attachment Theory and Close Relationships. Guilford Press, New York, NY US, pp. 46-76.

Campbell, L., Marshall, T., 2011. Anxious attachment and relationship processes: an interactionist perspective. J. Pers. 79, 917-947. 
Campbell, K.L., Schultheiss, O.C., McClelland, D.C., 1999. A necessary adjustment of protocol for use of DPC coat-A-count total testosterone assay with saliva. Clin. Biochem. 32, 83-85.

Carre, J.M., Iselin, A.-M.R., Welker, K.M., Hariri, A.R., Dodge, K.A., 2014. Testosterone reactivity to provocation mediates the effect of early intervention on aggressive behavior. Psychol. Sci. 25, 1140-1146.

Cassidy, J., Kobak, R.R., 1988. Avoidance and its relation to other defensive processes. In: Belsky, J., Nezworski, T. (Eds.), Clinical Implications of Attachment. Lawrence Erlbaum, Hillsdale, NJ, pp. 300-323.

Dabbs Jr., J.M., 1991. Salivary testosterone measurements: collecting, storing, and mailing saliva samples. Physiol. Behav. 49, 815-817.

Dabbs Jr., J.M., Hopper, C.H., Jurkovic, G.J., 1990. Testosterone and personality among college students and military veterans. Personal. Individ. Differ. 11, 1263-1269.

Deady, D., Smith, M., Sharp, M., Al-Dujaili, E., 2006. Maternal personality and reproductive ambition in women is associated with salivary testosterone levels. Biol. Psychol. 71, 29-32.

Del Giudice, M., Angeleri, R., 2016. Digit ratio (2D: 4D) and attachment styles in middle childhood: indirect evidence for an organizational effect of sex hormones. Adapt. Hum. Behav. Physiol. 2, 1-10.

Eagly, A.H., Wood, W., 1999. The origins of sex differences in human behavior: evolved dispositions versus social roles. Am. Psychol. 54, 408-423.

Edelstein, R.S., Chin, K., 2018. Hormones and close relationship processes: neuroendocrine bases of partnering and parenting. In: Schultheiss, O.C., Mehta, P.H. (Eds.), Routledge International Handbook of Social Neuroendocrinology. Routledge, Abingdon, UK, pp. 281-297.

Edelstein, R. S., et al., 2004. Adult attachment style and parental responsiveness during a stressful event. Attach Hum. Dev. 6, 31-52.

Edelstein, R.S., Chopik, W.J., Kean, E.L., 2011. Sociosexuality moderates the association between testosterone and relationship status in men and women. Horm. Behav. 60, 248-255.

Edelstein, R.S., Kean, E.L., Chopik, W.J., 2012. Women with an avoidant attachment style show attenuated estradiol responses to emotionally intimate stimuli. Horm. Behav. 61, 167-175.

Edelstein, R.S., van Anders, S.M., Chopik, W.J., Goldey, K.L., Wardecker, B.M., 2014. Dyadic associations between testosterone and relationship quality in couples. Horm. Behav. 65, 401-407.

Edelstein, R. S., et al., 2017. Prospective and dyadic associations between expectant parents' prenatal hormone changes and postpartum parenting outcomes. Dev. Psychobiol. 59, 77-90.

Endendijk, J. J., et al., 2016. Diurnal testosterone variability is differentially associated with parenting quality in mothers and fathers. Horm. Behav. 80, 68-75. 
Fleming, A.S., Corter, C., Stallings, J., Steiner, M., 2002. Testosterone and prolactin are associated with emotional responses to infant cries in new fathers. Horm. Behav. 42, 399-413.

Gettler, L.T., McDade, T.W., Agustin, S.S., Kuzawa, C.W., 2011a. Short-term changes in fathers' hormones during father-child play: impacts of paternal attitudes and experience. Horm. Behav. 6o, 599-606.

Gettler, L.T., McDade, T.W., Feranil, A.B., Kuzawa, C.W., 2011b. Longitudinal evidence that fatherhood decreases testosterone in human males. Proc. Natl. Acad. Sci. U. S. A. 108, 16194-16199.

Granger, D.A., Shirtcliff, E.A., Booth, A., Kivlighan, K.T., Schwartz, E.B., 2004. The "trouble" with salivary testosterone. Psychoneuroendocrinology 29, 1229-1240.

Gray, P.B., Parkin, J.C., Samms-Vaughan, M.E., 2007. Hormonal correlates of human paternal interactions: a hospital-based investigation in urban Jamaica. Horm. Behav. 52, 499-507.

Harris, J.A., Rushton, J.P., Hampson, E., Jackson, D.N., 1996. Salivary testosterone and self-report aggressive and pro-social personality characteristics in men and women. Aggress. Behav. 22, 321-331.

John, O.P., Naumann, L.P., Soto, C.J., 2008. Paradigm shift to the integrative big five trait taxonomy. In: John, O.P. (Ed.), Handbook of Personality: Theory and Research. Guilford, New York, NY, pp. 114-158.

Kaiser, H., Powers, S., 2006. Testosterone and conflict tactics within lateadolescent couples: a dyadic predictive model. J. Soc. Pers. Relat. 23, 231-248.

Keevil, B.G., et al., 2017. Distribution of salivary testosterone in men and women in a British general population-based sample: the third national survey of sexual attitudes and lifestyles (Natsal-3). J. Endocr. Soc. 1, 14-25.

Kenny, D.A., Kashy, D.A., Cook, W.L., 2006. Dyadic Data Analysis. The Guilford Press, New York, NY.

Khan-Dawood, F.S., Choe, J.K., Dawood, M.Y., 1984. Salivary and plasma bound and "free" testosterone in men and women. Am. J. Obstet. Gynecol. 148, 441-445.

Kuo, P.X., Saini, E.K., Thomason, E., Schultheiss, O.C., Gonzalez, R., Volling, B.L., 2016. Individual variation in fathers' testosterone reactivity to infant distress predicts parenting behaviors with their 1-year-old infants. Dev. Psychobiol. 58, 303-314. http://digitalcommons.unl.edu/famconfacpub/251

Kuo, P. X., et al., 2018. Fathers' cortisol and testosterone in the days around infants' births predict later paternal involvement. Horm. Behav. 106, 28-34. http://digitalcommons.unl.edu/famconfacpub/255

Liening, S.H., Stanton, S.J., Saini, E.K., Schultheiss, O.C., 2010. Salivary testosterone, cortisol, and progesterone: two-week stability, interhormone correlations, and effects of time of day, menstrual cycle, and oral contraceptives use on steroid hormone levels. Physiol. Behav. 99, 8-16.

Maestripieri, D., Baran, N.M., Sapienza, P., Zingales, L., 2010. Between-and within-sex variation in hormonal responses to psychological stress in a large sample of college students. Stress Int. J. Biol. Stress 5, 413-424. 
Magrini, G., Chiodoni, G., Rey, F., Felber, J.P., 1986. Further evidence for the usefulness of the salivary testosterone radioimmunoassay in the assessment of androgenicity in man in basal and stimulated conditions. Horm. Res. Paediatr. $23,65-73$.

Mascaro, J.S., Hackett, P.D., Rilling, J.K., 2013. Testicular volume is inversely correlated with nurturing-related brain activity in human fathers. Proc. Natl. Acad. Sci. U.S.A. 110, 15746-15751.

McCrae, R.R., John, O.P., 1992. An introduction to the five-factor model and its applications. J. Pers. 6o, 175-215.

Mehta, P.H., Prasad, S., 2015. The dual-hormone hypothesis: a brief review and future research agenda. Curr. Opin. Behav. Sci. 3, 163-168.

Mikulincer, M., Gillath, O., Shaver, P.R., 2002. Activation of the attachment system in adulthood: threat-related primes increase the accessibility of mental representations of attachment figures. J. Pers. Soc. Psychol. 83, 881-895.

Noftle, E.E., Shaver, P.R., 2006. Attachment dimensions and the big five personality traits: associations and comparative ability to predict relationship quality. J. Res. Pers. 40, 179-208.

Raisanen, J.C., Chadwick, S.B., Michalak, N., van Anders, S.M., 2018. Average associations between sexual desire, testosterone, and stress in women and men over time. Arch. Sex. Behav. 47, 1613-1631.

Rholes, W.S., Simpson, J.A., Blakely, B.S., Lanigan, L., Allen, E.A., 1997. Adult attachment styles, the desire to have children, and working models of parenthood. J. Pers. 65, 357-385.

Rholes, W.S., Simpson, J.A., Campbell, L., Grich, J., 2001. Adult attachment and the transition to parenthood. J. Pers. Soc. Psychol. 81, 421-435.

Rholes, W.S., Simpson, J.A., Friedman, M., 2006. Avoidant attachment and the experience of parenting. Personal. Soc. Psychol. Bull. 32, 275-285.

Safyer, P., Volling, B.L., Schultheiss, O.C., Tolman, R.M., 2019. Adult attachment, implicit motives, and mothers' and fathers' parenting behaviors. Motiv. Sci.

5(3), 220-234.

Sankar, J.S., 2015. The Role of Androgens in Life History Theories of Attachment. (Unpublished doctoral dissertation).

Sapolsky, R.M., 1987. Stress, social status, and reproductive physiology in freeliving baboons. In: Crews, D. (Ed.), Psychobiology and Reproductive Behavior: An Evolutionary Perspective. Prentice-Hall, Englewood Cliffs, NJ, pp. 291-322.

Saxbe, D.E., et al., 2017. Fathers' decline in testosterone and synchrony with partner testosterone predicts greater postpartum relationship investment. Horm. Behav. 90, 39-47.

Schultheiss, O.C., Stanton, S.J., 2009. Assessment of salivary hormones. In: Harmon- Jones, E., Beer, J.S. (Eds.), Methods in Social Neuroscience. Guilford Press, New York, pp. 17-44.

Sellers, J.G., Mehl, M.R., Josephs, R.A., 2007. Hormones and personality: testosterone as a marker of individual differences. J. Res. Pers. 41, 126-138. 
Shaver, P.R., Mikulincer, M., 2007. Attachment theory and research: core concepts, basic principles, conceptual bridges. In: Kruglanski, A.W., Higgins, E.T. (Eds.), Social Psychology: Handbook of Basic Principles, 2nd ed. Guilford Press, New York, NY, pp. 650-677.

Shirtcliff, E.A., Granger, D.A., Likos, A., 2002. Gender differences in the validity of testosterone measured in saliva by immunoassay. Horm. Behav. 42, 62-69.

Simpson, J.A., Rholes, S.W., 2012. Adult attachment orientations, stress, and romantic relationships. In: Devine, P.G., Plant, E.A. (Eds.), Advances in Experimental Social Psychology. Academic Press, Burlington, pp. 279-328.

Simpson, J.A., Rholes, W.S., Phillips, D., 1996. Conflict in close relationships: an attachment perspective. J. Pers. Soc. Psychol. 71, 899-914.

Simpson, J.A., Rholes, W.S., Campbell, L., Tran, S., Wilson, C.L., 2003. Adult attachment, the transition to parenthood, and depressive symptoms. J. Pers. Soc. Psychol. 84, 1172-1187.

Smeets-Janssen, M. M., et al., 2015. Salivary testosterone is consistently and positively associated with extraversion: results from the Netherlands study of depression and anxiety. Neuropsychobiology. 71, 76-84.

Soler, H., Vinayak, P., Quadagno, D., 2000. Biosocial aspects of domestic violence. Psychoneuroendocrinology 25, 721-739.

Storey, A.E., Walsh, C.J., Quinton, R.L., Wynne-Edwards, K.E., 200o. Hormonal correlates of paternal responsiveness in new and expectant fathers. Evol. Hum. Behav. 21, 79-95.

Storey, A.E., Noseworthy, D.E., Delahunty, K.M., Halfyard, S.J., McKay, D.W., 2011. The effects of social context on the hormonal and behavioral responsiveness of human fathers. Horm. Behav. 6o, 353-361.

Turan, B., Guo, J., Boggiano, M.M., Bedgood, D., 2014. Dominant, cold, avoidant, and lonely: basal testosterone as a biological marker for an interpersonal style. J. Res. Pers. 50, 84-89.

van Anders, S.M., Goldey, K.L., 2010. Testosterone and partnering are linked via relationship status for women and 'relationship orientation' for men. Horm. Behav. 58, 820-826.

van Anders, S.M., Hamilton, L.D., Watson, N.V., 2007. Multiple partners are associated with higher testosterone in North American men and women. Horm. Behav. 51, 454-459.

van Anders, S.M., Brotto, L., Farrell, J., Yule, M., 2009. Associations among physiological and subjective sexual response, sexual desire, and salivary steroid hormones in healthy premenopausal women. J. Sex. Med. 6, 739-751.

van Anders, S.M., Goldey, K.L., Kuo, P.X., 2011. The Steroid/Peptide Theory of Social Bonds: Integrating testosterone and peptide responses for classifying social behavioral contexts. Psychoneuroendocrinology 36, 1265-1275. http:// digitalcommons.unl.edu/famconfacpub/257

Volling, B.L., Gonzalez, R., Oh, W., Song, J., Yu, T., Rosenberg, L., Kuo, P.X., Thomason, E., Beyers-Carlson, E., Safyer, P., Stevenson, M.M., 2017. Developmental trajectories of children's adjustment across the transition to 
siblinghood: Pre-birth predictors and sibling outcomes at one year. Monogr.

Soc. Res. Child Dev. 82 (3), 1-216.

Voorthuis, A., Bakermans-Kranenburg, M., van IJzendoorn, M., 2019. Testosterone reactivity to infant crying and caregiving in women: the role of oral contraceptives and basal cortisol. Infant Behav. Dev. 56, 101191.

Wardecker, B.M., Chopik, W.J., Moors, A.C., Edelstein, R.S., 2019. Avoidant attachment style. In: Zeigler-Hill, V., Shackelford, T.K. (Eds.), Encyclopedia of Personality and Individual Differences. Springer (in press).

Weisman, O., Zagoory-Sharon, O., Feldman, R., 2014. Oxytocin administration, salivary testosterone, and father-infant social behavior. Prog. NeuroPsychopharmacol. Biol. Psychiatry 49, 47-52.

Wingfield, J.C., Hegner, R.E., Dufty, A.M., Ball, G.F., 1990. The "challenge hypothesis": theoretical implications for patterns of testosterone secretion, mating systems, and breeding strategies. Am. Nat. 136, 829-846.

Zilioli, S., Bird, B.M., 2017. Functional significance of men's testosterone reactivity to social stimuli. Front. Neuroendocrinol. 47, 1-18. 